\title{
Angular and Energy Distributions of Electrons Produced in Arbitrary Biomaterials by Proton Impact
}

\author{
Pablo de Vera, ${ }^{1, *}$ Rafael Garcia-Molina, ${ }^{2}$ and Isabel Abril ${ }^{1}$ \\ ${ }^{1}$ Departament de Física Aplicada, Universitat d'Alacant, E-03080 Alacant, Spain \\ ${ }^{2}$ Departamento de Física-Centro de Investigación en Óptica y Nanofísica, Regional Campus of International Excellence \\ "Campus Mare Nostrum," Universidad de Murcia, E-30100 Murcia, Spain
}

(Received 28 July 2014; published 5 January 2015)

\begin{abstract}
We present a simple method for obtaining reliable angular and energy distributions of electrons ejected from arbitrary condensed biomaterials by proton impact. Relying on a suitable description of the electronic excitation spectrum and a physically motivated relation between the ion and electron scattering angles, it yields cross sections in rather good agreement with experimental data in a broad range of ejection angles and energies, by only using as input the target composition and density. The versatility and simplicity of the method, which can be also extended to other charged particles, make it especially suited for obtaining ionization data for any complex biomaterial present in realistic cellular environments.
\end{abstract}

DOI: 10.1103/PhysRevLett.114.018101

PACS numbers: 87.53.-j, 78.70.-g, 79.20.-m, 87.14.gk

A precise knowledge of the angular and energy distribution of secondary electrons ejected from biological materials after swift ion impact is a matter of paramount importance in the development of current radiobiological models aimed at understanding the mechanisms underlying ion beam cancer therapy. It is well known that energetic ions have a characteristic depth-dose curve, with a sharp maximum at the end of their trajectories (the so-called Bragg peak), where the ionization efficiency is maximum. This fact makes ion beam cancer therapy one of the most outstanding techniques for oncological treatment nowadays $[1,2]$, due to the concentration of the radiation-induced damage in the desired tumor volumes, while sparing the healthy surrounding regions. Among the different phenomena involved in the multiscale mechanism of ion beam-induced biodamage [3], production of secondary electrons is especially relevant $[4,5]$, since they transport through nanometer to micrometer distances the energy lost by the projectile around its track, giving place to very sharp and intense radial dose distributions $[6,7]$. This fact explains the increased radiobiological efficiency of ions compared with photons, for which the microscopic patterns of dose deposition are much more homogeneous $[2,8]$. Moreover, it has been shown in recent years that very lowenergy electrons are responsible for especially harmful interactions in DNA (and other biological targets), such as dissociative electron attachment [9-11]. Thus, an accurate knowledge of the spatial and energetic distribution of electrons around ion tracks becomes crucial, particularly near the Bragg peak, where most of the biodamage is expected to take place.

A realistic calculation of track-structure-dependent magnitudes in biological targets needs as input both the energy and angular distributions of electrons produced by the incoming ions. Although these spectra are reasonably well known for water [12-16], it is necessary to accurately know them for a great variety of organic materials present in cells $[17,18]$, and for a wide range of incident ion and secondary electron energies. For these purposes, several existing theoretical models can be used to calculate these distributions. For example, quite simple methods such as the binary encounter approximation [19] or the Rudd model [20] yield electron energy spectra with reasonable accuracy, but for a limited range of energies and some selected targets [4]. Moreover, in some cases the angular distributions of ejected electrons are not provided. More sophisticated and very accurate methods exist, such as the $a b$ initio continuum distorted wave (CDW) and continuum distorted wave eikonal initial state (CDW-EIS) methods [17,21-24], which can also be applied to arbitrary biomolecules [17]; nonetheless, their use for very large macromolecules or condensed phase targets (such as those found in biological environments) requires some computational efforts that can be reduced by using analytical expressions, as proposed in this work.

As an alternative, the dielectric formalism $[25,26]$ has been also used to calculate the angular and energy distributions with reasonable accuracy [13,27]. Nonetheless, these analyses have so far been restricted to water, and some methodologies need to evaluate the wave functions of the escaping electrons [27], which complicates the calculations, while others make use of a number of empirical approximations [13].

It is also possible to use the dielectric formalism, together with physically motivated approximations and a suitable description of the electronic excitation spectrum of biological materials, to obtain accurate electron energy spectra and total ionization cross sections (TICS) for ion impact in arbitrary condensed biological materials, by only knowing their atomic composition and density [28]. Since the spectrum of the electronic excitations depends both on their energy and momentum, the dielectric formalism is 
able to produce not only energy distributions [i.e., singly differential cross sections (SDCS)], but also angular distributions [i.e., doubly differential cross sections (DDCS)] of secondary electrons, which are linked to the momentum transferred by incident projectiles.

The aim of this Letter is to develop a method to calculate angular, as well as energy, distributions of secondary electrons (DDCS) emitted from arbitrary biomaterials impacted by swift protons, using only a minimum of essential information about the biological target (composition and density). An essential point of this work is the proposal of a relation between the momentum transfer and the ejection angle of secondary electrons produced by proton impact.

Based on the dielectric formalism [25,26], we write the DDCS for ionizing an electronic shell $j$ as a function of the secondary electron energy $W$ and the momentum transfer to the target $k$ as $[28,29]$

$$
\left.\frac{d^{2} \sigma}{d W d k}\right|_{j}=\frac{e^{2}}{\pi \hbar^{2} \mathcal{N}} \frac{M[Z-\rho(k)]^{2}}{T} \frac{1}{k} \operatorname{Im}\left[\frac{-1}{\epsilon\left(k, B_{j}+W\right)}\right]_{j}
$$

where $T, M$, and $Z$ are, respectively, the kinetic energy, mass, and atomic number of the projectile, and $\rho(k)$ is the Fourier transform of its electronic density. The target is characterized by its molecular density $\mathcal{N}$ and the energy loss function (ELF), $\operatorname{Im}[-1 / \epsilon(k, E)]$, which represents its electronic excitation spectrum; $E=B_{j}+W$ is the transferred energy in an ionizing collision, $B_{j}$ being the binding energy of a given electronic inner shell, or the mean binding energy of the outer shell electrons $[28,30]$. The subscript $j$ in the ELF refers to the contribution coming from the specific shell $j$. The ELF is usually obtained from experimental data in the optical limit $(k=0)$, being extended to arbitrary values of the momentum transfer through different dispersion relations [31]. We will use here the Mermin energy loss function (MELF) generalized oscillator strength (GOS) method [32], which employs Mermin dielectric functions [33] for describing the outer-shell electron excitations, while inner shells are accounted for by hydrogenic generalized oscillator strengths [34]. By making use of this procedure the experimental ELF of liquid water [35] and other materials is accurately reproduced over the whole energy and momentum transfers plane [36].

It is straightforward to rewrite the $\operatorname{DDCS}(W, k)$ in Eq. (1) as a function of the scattering angle $\phi$ of the projectile by using its relation with the momentum transfer,

$$
k=\sqrt{\left(2 M / \hbar^{2}\right)(2 T-E-2 \sqrt{T(T-E)} \cos \phi)} .
$$

In order to obtain the $\operatorname{DDCS}(W, \theta)$ as a function of the ejected angle $\theta$ of the secondary electron, we will assume that both angles are related by $\phi=\alpha \theta$. The proportionality constant $\alpha$ can be obtained by bearing in mind that the $\operatorname{DDCS}(W, \theta)$ for ejection of electrons is dominated by the so-called binary encounter peak, located at $\theta^{\mathrm{BE}}=$ $\arccos (\sqrt{W /(4 \mu T)})$ [20], at which the collision process can be regarded as a binary collision between free particles; $\mu=m / M$, where $m$ is the mass of the electron. The $\operatorname{DDCS}(W, \phi)$ as a function of the scattering angle of the projectile is also dominated by a maximum, at an angle that we will call $\phi^{\mathrm{max}}$. If this maximum also corresponds to the binary peak angle, given by $\phi^{\mathrm{BE}}=$ $\arctan \left\{\left[\mu \sin \left(\pi-2 \theta^{\mathrm{BE}}\right)\right] /\left[1+\mu \cos \left(\pi-2 \theta^{\mathrm{BE}}\right)\right]\right\}$, then $\alpha$ can be obtained analytically by only considering the kinematics of the binary collision; note that $\phi$ always takes very small values, so $\alpha$ will be $\sim 10^{-4}$ to yield appropriate values for $\theta$.

To test this hypothesis, we have plotted in Fig. 1 the position of $\phi^{\mathrm{BE}}$ as a function of $W$, for $1 \mathrm{MeV}$ and $10 \mathrm{MeV}$ protons. The $\phi^{\max }$ values obtained from the $\operatorname{DDCS}(W, \phi)$ using the MELF-GOS fitting to the experimental ELF of liquid water [35] are also shown in Fig. 1. It can be seen that both angles are very similar, and have the same behavior. Therefore, as $\phi^{\max } \simeq \phi^{\mathrm{BE}}$, we conclude that the maximum in $\operatorname{DDCS}(W, \phi)$ also corresponds to the binary encounter peak, so we will consider that the proportionality constant relating $\phi$ and $\theta$ can be obtained analytically as

$$
\alpha=\phi^{\mathrm{BE}} / \theta^{\mathrm{BE}} .
$$

Although the proportionality constant $\alpha$ is obtained from the binary encounter peak angle, we assume that it is also valid for all the $\theta$ range. Proceeding in this way, $\alpha$ provides the correct position of the binary peak in the angular spectrum, while its structure is determined by the momentum transfer distribution accounted for by the electronic excitation spectrum of the target through its ELF.

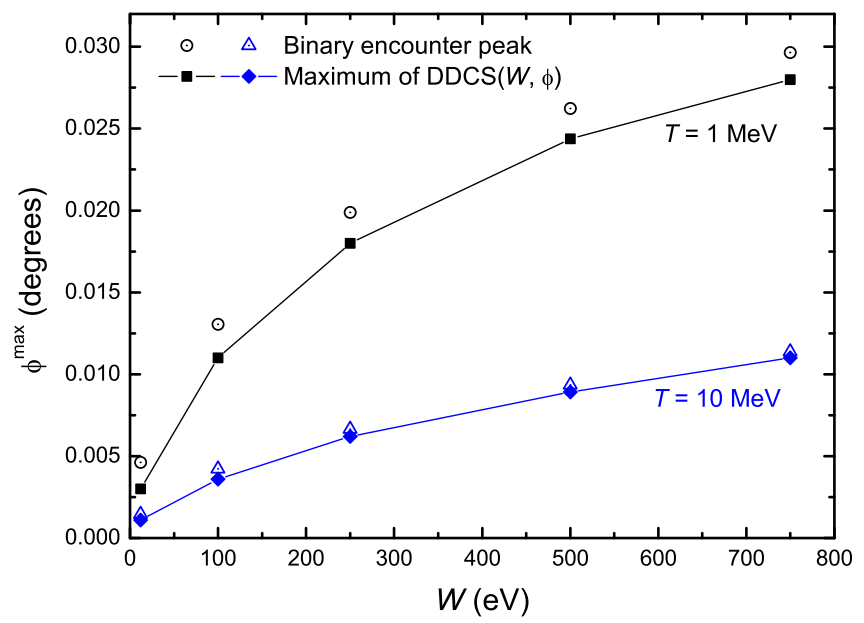

FIG. 1 (color online). Behavior of $\phi^{\max }$ as a function of the energy $W$ of the ejected electron, for protons incident in liquid water with $T=1 \mathrm{MeV}$ and $10 \mathrm{MeV}$. The lines are drawn to guide the eye. 
By making use of Eq. (2) and the definition of the solid angle for the ejected electron, $d \Omega=2 \pi \sin \theta d \theta$, we obtain from Eq. (1) the following expression:

$$
\begin{aligned}
\left.\frac{d^{2} \sigma}{d W d \Omega}\right|_{j}= & \frac{\alpha e^{2}}{2 \pi^{2} \hbar^{2} \mathcal{N} \sin \theta} \frac{M[Z-\rho(k)]^{2}}{T} \operatorname{Im}\left[\frac{-1}{\epsilon\left(k, B_{j}+W\right)}\right]_{j} \\
& \times \frac{\sqrt{T\left(T-\left(B_{j}+W\right)\right)} \sin (\alpha \theta)}{2 T-\left(B_{j}+W\right)-2 \sqrt{T\left(T-\left(B_{j}+W\right)\right)} \cos (\alpha \theta)},
\end{aligned}
$$

which is the $\operatorname{DDCS}(W, \theta)$ for ejecting electrons from the $j$ shell in the angle $\theta$, per unit energy and solid angle. In order to check the feasibility of this expression, we compare its results with the experimental $\operatorname{DDCS}(W, \theta)$ for proton impact in water vapor [37]. Since data for liquid water are not available, we use the gas phase data just to check the general performance of the method. Figure 2(a) shows our calculations by dashed lines, together with experimental data depicted by symbols, for $300 \mathrm{keV}$ protons in water, as a function of the ejection angle, for two kinetic energies of the escaping electrons, $W=9.6 \mathrm{eV}$ and $W=200 \mathrm{eV}$. As can be seen, the main characteristics of the calculations (order of magnitude and features) are in qualitatively good agreement with the experimental data. The meaning of the other curves will be discussed in what follows, where further improvements will be introduced.

Given the simple assumption upon which Eq. (3) is based, two corrections can be introduced to achieve a better agreement with the experiments. First of all, it is well known that the first Born approximation (on which the dielectric formalism is based) is not very accurate for calculating DDCS, since it does not take into account two and three center effects (the ejected electron being attracted by the projectile and/or the residual ion after the collision). Such a situation can be consistently accounted for by CDW calculations [21]. Within the dielectric formalism, in which the present calculations are based, the number of electrons ejected in the forward direction, attracted by the field of the projectile after the collision (a process known as electron capture to the continuum), has to be semiempirically corrected multiplying Eq. (3) by the so-called Salin's factor $[13,20,40]$. Dotted lines in Fig. 2(a) represent the results of Eq. (3) when the Salin's factor is taken into account, showing an improvement in the results for $200 \mathrm{eV}$ electrons in the forward direction (i.e., small angles $\theta$ ).

The discrepancy observed for $W=9.6 \mathrm{eV}$ could come from the small differences between $\phi^{\mathrm{BE}}$ and $\phi^{\mathrm{max}}$ observed in Fig. 1, which are more relevant (in relative terms) at low ejection energies. Therefore, an obvious correction might be to replace $\phi^{\mathrm{BE}}$ by $\phi^{\mathrm{max}}$ in Eq. (2), i.e., $\alpha=\phi^{\mathrm{max}} / \theta^{\mathrm{BE}}$. We will refer to this as the "peak tuning" correction. The fully improved calculations obtained with Eq. (3) when using Salin's factor and the peak tuning correction are depicted by thick solid lines in Fig. 2(a). Now the agreement between the

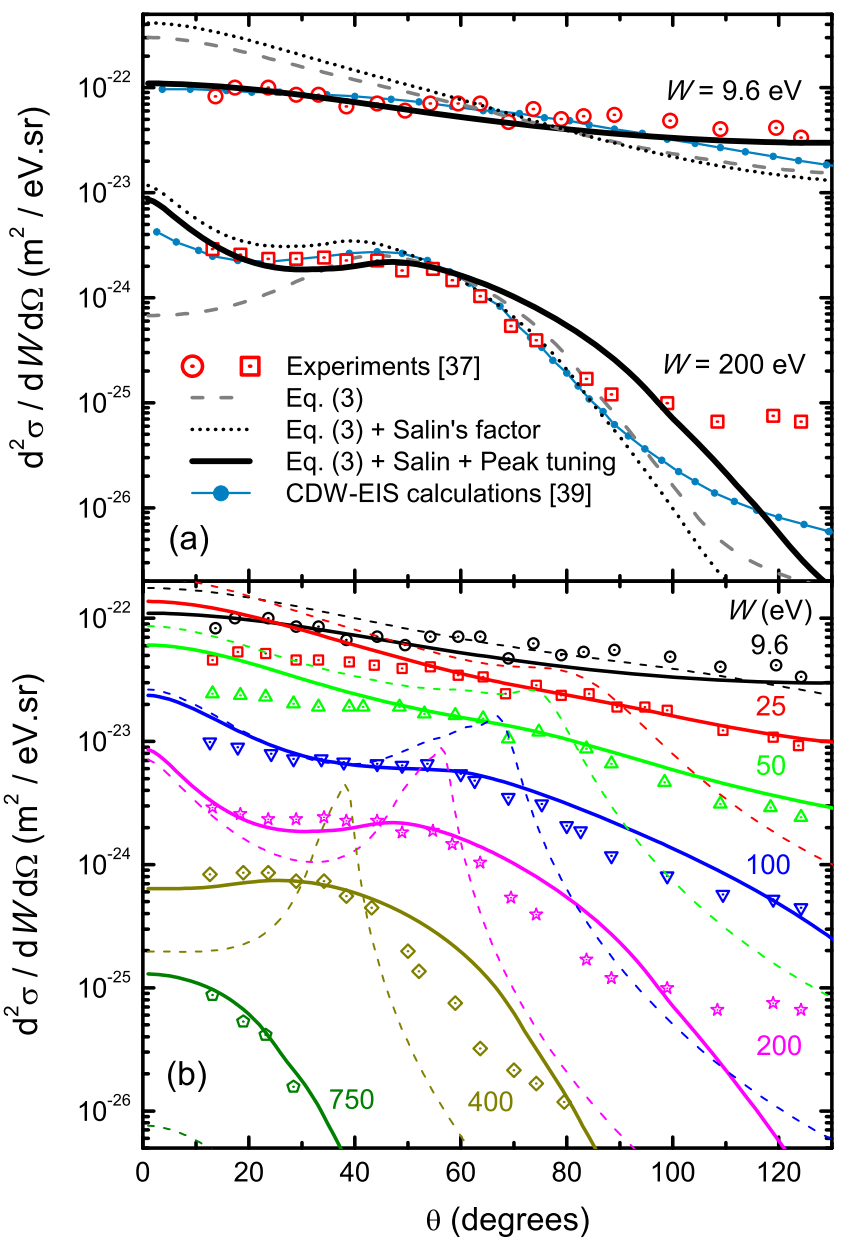

FIG. 2 (color online). Comparison of calculated and measured DDCS [37] as a function of the ejected electron angle $\theta$, for the case of $300 \mathrm{keV} \mathrm{H} \mathrm{H}^{+}$incident on water. (a) Results for $W=$ $9.6 \mathrm{eV}$ and $200 \mathrm{eV}$ obtained with Eq. (3) (dashed line), and further improvements, as discussed in the text (dotted and solid lines). The solid lines with symbols represent CDW-EIS calculations [38]. (b) Results for all the ejection energies reported in Ref. [37] are shown by symbols. Curves are our fully improved calculations obtained when the electronic excitation spectrum of liquid water is described using the MELF-GOS method (solid lines) [32] or the extended Drude method (dashed lines) [39].

calculations and the experiments is rather good for both ejection energies and in a wide range of ejection angles. For comparison, we also show in Fig. 2(a) CDW-EIS calculations [38] by solid lines with symbols. It can be seen that our results are quite similar to these $a b$ initio calculations, despite the simplicity of our methodology with respect these quantum calculations, as well as the possible phase effects.

Figure 2(b) shows, by solid lines, the calculations obtained with the previous prescription [Eq. (3) with Salin's factor and peak tuning correction] for all the secondary electron energies reported in the experiment for $300 \mathrm{keV} \mathrm{H}^{+}$impact in water [37], which are depicted by symbols. The good agreement holds for this broader range of ejected electron energies $W$. 
The dashed curves in Fig. 2(b) are plotted to discuss the role played by a proper description of the target electronic excitation spectrum through its ELF, as it appears in Eq. (3). The solid curves were obtained by using the MELF-GOS method [32], where the extension to $k \neq 0$ values is automatically accounted for by the analytical properties of the Mermin ELF [33]. Nonetheless, a widely employed extension algorithm (referred to as the extended Drude method [39]) consists of a quadratic $k$ dependence of the excitation spectrum resonance energies, which leads to substantial differences from the MELF-GOS results $[23,31,36]$. The DDCS obtained with the extended Drude method, depicted by dashed curves in Fig. 2(b), cannot reproduce the experimental data, due to the simpler $k \neq 0$ extension algorithm. Therefore, it is clear that a suitable description of the target electronic excitation spectrum over the whole momentum and energy transfer surface, through its ELF, is essential for obtaining angular distributions of secondary electrons in good agreement with experimental data.

An advantage of the proposed model, besides its simplicity, is its predictive power, since it can easily be applied to any biological material for which the ELF is provided, either experimentally, theoretically or semiempirically. This is particularly relevant for radiobiological models, which need the angular and energetic distributions of secondary electrons generated in realistic biomaterials around the tracks of swift ions, as the ones currently used in hadron therapy [1] or unavoidably present in manned space missions [41]. Although there are no experimental data for all the relevant biological materials, it is possible to predict their ELF in the optical limit making use of an empirical parametrization [42], which only needs as input the atomic composition and density of the material. The feasibility of this parametrization to predict the SDCS and TICS of biomaterials with good accuracy has been already demonstrated [28]. Therefore, the methodology proposed in this work, together with the parametric approach for obtaining the optical ELF, virtually allows us to obtain DDCS, as well as SDCS and TICS, for electrons generated by swift ions incident on any biological material (independently of its complexity).

To illustrate the capabilities of the present procedure, Fig. 3 shows the DDCS for electrons generated by $1 \mathrm{MeV}$ proton impact in the DNA base adenine, as a function of their ejection angle [Fig. 3(a)] and energy [Fig. 3(b)]. Solid lines are calculations using the experimental optical ELF of solid adenine [43] extended to $k \neq 0$ by the MELF-GOS method, while symbols represent experimental data in the gas phase [44]. The agreement is rather good, especially in Fig. 3(b), taking into account the simplicity of the method and the possible experimental uncertainties and phase effects. Since the value of $\alpha$ has been obtained from the BE peak assumption of Eq. (2), the results presented in this work should be restricted to ejection angles far from the backward direction. Nonetheless, as the DDCS are dominated by this peak, vanishing at high angles and energies, the present approximation yields good results over the angular range
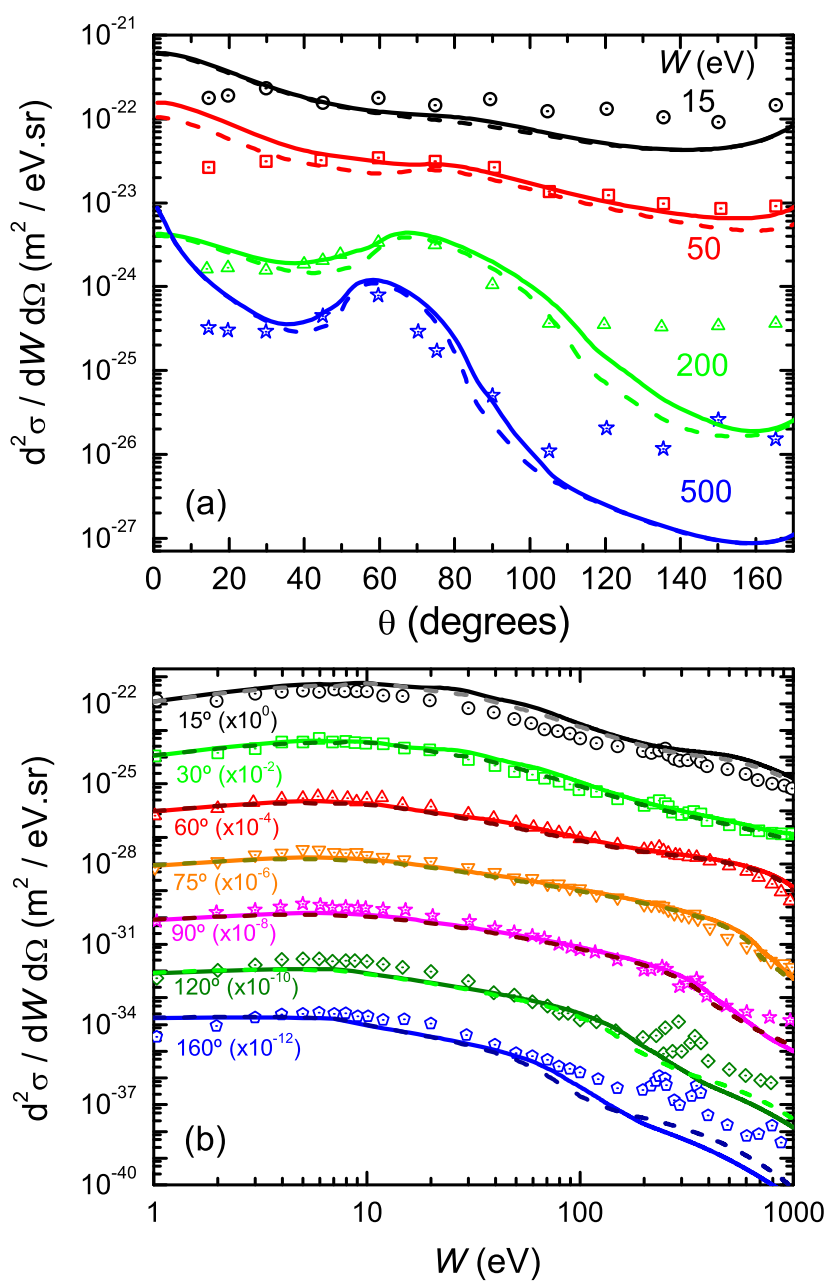

FIG. 3 (color online). DDCS of secondary electrons generated by $1 \mathrm{MeV} \mathrm{H}^{+}$in adenine as a function of (a) $\theta$ and (b) $W$. Symbols are experimental data for the gas phase [44], while lines are present calculations for solid adenine based on the experimental ELF (solid line) or the parametrized one (dashed lines).

where the DDCSs are more significant. The peaked structures appearing at high $W$ and $\theta$ values are due to Auger electrons, which are not accounted for in the present calculations, although they could be [45], since the probability of ionizing inner shells can be calculated.

Although we have used the experimentally available optical ELF of adenine, in order to show the predictive power of the method we also show in Fig. 3 by dashed lines the results obtained from the ELF provided by the empirical parametric model [42]. It can be seen that the results are in a similar level of accuracy, a fact which is very promising taking into account the possibility of calculating DDCS even when experimental information for the electronic excitation spectrum of the target is lacking.

In conclusion, we have presented a method to obtain analytically the energy and angular dependence of the DDCS for electrons ejected after proton impact on any kind of biological target. This procedure relies on the proposed relation between the scattering angle of the incident ion and the ejection angle of the secondary electron, which is 
obtained by making physically motivated approximations, and supported by the good agreement of our results with the available experimental data. Because this method is based on the dielectric formalism, the only needed input is the electronic excitation spectrum of the target through its optical ELF (which can be obtained either from experimental data or by a semiempirical parametrization), properly extended to $k \neq 0$. Due to its simplicity, versatility, and predictive power, the method proposed in this work is especially suited for calculating differential ionization cross sections in processes involved in ion beam damage in realistic cellular environments. Also, this methodology can be easily extended for other charged particles, such as heavier ions or electrons, by simply accounting for electron exchange effects or the ion charge state in Eq. (3).

The authors recognize the financial support from the Spanish Ministerio de Economía y Competitividad and the European Regional Development Fund (Project No. FIS2010-17225). PdV acknowledges financial support from the European Union's FP7-People Program (Marie Curie Actions) within the Initial Training Network No. 608163 "ARGENT". Support from the European COST Action MP1002 NanoIBCT is gratefully acknowledged.

*pablo.vera@ua.es

[1] J. S. Loeffler and M. Durante, Nature Rev. Clin. Oncol. 10, 411 (2013).

[2] D. Schardt, T. Elsässer, and D. Schulz-Ertner, Rev. Mod. Phys. 82, 383 (2010).

[3] A. V. Solov'yov, E. Surdutovich, E. Scifoni, I. Mishustin, and W. Greiner, Phys. Rev. E 79, 011909 (2009).

[4] M. E. Rudd, Y.-K. Kim, T. Märk, J. Schou, N. Stolterfoht, and L. H. Toburen, Secondary Electron Spectra from Charged Particle Interactions (International Commission on Radiation Units and Measurements, Bethesda, MD, 1996).

[5] Dz. Belkić, J. Math. Chem. 47, 1366 (2010).

[6] E. Surdutovich, D. C. Gallagher, and A. V. Solov'yov, Phys. Rev. E 84, 051918 (2011).

[7] H. Nikjoo, S. Uehara, W. E. Wilson, M. Hoshi, and D. T. Goodhead, Int. J. Radiat. Biol. 73, 355 (1998).

[8] M. Scholz and G. Kraft, Adv. Space Res. 18, 5 (1996).

[9] B. Boudaïffa, P. Cloutier, D. Hunting, M. A. Huels, and L. Sanche, Science 287, 1658 (2000).

[10] H. Abdoul-Carime, S. Gohlke, and E. Illenberger, Phys. Rev. Lett. 92, 168103 (2004).

[11] C. König, J. Kopyra, I. Bald, and E. Illenberger, Phys. Rev. Lett. 97, 018105 (2006).

[12] F. Gobet, B. Farizon, M. Farizon, M. J. Gaillard, M. Carré, M. Lezius, P. Scheier, and T. D. Märk, Phys. Rev. Lett. 86, 3751 (2001).

[13] M. Dingfelder, Radiat. Prot. Dosim. 99, 23 (2002).

[14] C. Champion and C. Dal Cappello, Nucl. Instrum. Methods Phys. Res., Sect. B 267, 881 (2009).

[15] C. Dal Cappello, C. Champion, O. Boudrioua, H. Lekadir, Y. Sato, and D. Ohsawa, Nucl. Instrum. Methods Phys. Res., Sect. B 267, 781 (2009).
[16] S. Nandi, S. Biswas, A. Khan, J. M. Monti, C. A. Tachino, R. D. Rivarola, D. Misra, and L. C. Tribedi, Phys. Rev. A 87, 052710 (2013).

[17] M. E. Galassi, C. Champion, P. F. Weck, R. D. Rivarola, O. Fojón, and J. Hanssen, Phys. Med. Biol. 57, 2081 (2012).

[18] A. N. Agnihotri, S. Nandi, S. Kasthurirangan, A. Kumar, M. E. Galassi, R. D. Rivarola, C. Champion, and L. C. Tribedi, Phys. Rev. A 87, 032716 (2013).

[19] L. Vriens, Proc. Phys. Soc. London 90, 935 (1967).

[20] M. E. Rudd, Y.-K. Kim, D. H. Madison, and T. J. Gay, Rev. Mod. Phys. 64, 441 (1992).

[21] Dz. Belkić, J. Phys. B 11, 3529 (1978).

[22] D. S. F. Crothers and J. F. McCann, J. Phys. B 16, 3229 (1983).

[23] Fast Ion-Atom and Ion-Molecule Collisions, edited by Dz. Belkić (World Scientific, Singapore, 2012).

[24] Theory of Heavy Ion Collision Physics in Hadron Therapy, Advances in Quantum Chemistry 65, edited by Dz. Belkić (Elsevier, New York, 2013).

[25] J. Lindhard, K. Dan. Vidensk. Selsk. Mat. Fys. Medd. 28, 8 (1954).

[26] R. H. Ritchie, Phys. Rev. 106, 874 (1957).

[27] K. A. Long and G. J. Paretzke, J. Chem. Phys. 95, 1049 (1991).

[28] P. de Vera, R. Garcia-Molina, I. Abril, and A. V. Solov'yov, Phys. Rev. Lett. 110, 148104 (2013).

[29] H. Nikjoo, S. Uehara, and D. Emfietzoglou, Interaction of Radiation with Matter (CRC Press, Boca Raton, 2012), Chap. 19.

[30] P. de Vera, I. Abril, R. Garcia-Molina, and A. V. Solov'yov, J. Phys. Conf. Ser. 438, 012015 (2013).

[31] R. Garcia-Molina, I. Abril, I. Kyriakou, and D. Emfietzoglou, Radiation Damage in Biomolecular Systems, edited by G. García Gómez-Tejedor and M. C. Fuss (Springer, Dordrecht, 2012), Chap. 15.

[32] S. Heredia-Avalos, R. Garcia-Molina, J. M. FernándezVarea, and I. Abril, Phys. Rev. A 72, 052902 (2005).

[33] N. D. Mermin, Phys. Rev. B 1, 2362 (1970).

[34] R. F. Egerton, Electron Energy-Loss Spectroscopy in the Electron Microscope (Plenum, New York, 1989).

[35] H. Hayashi, N. Watanabe, Y. Udagawa, and C. C. Kao, Proc. Natl. Acad. Sci. U.S.A. 97, 6264 (2000).

[36] I. Abril, C. D. Denton, P. de Vera, I. Kyriakou, D. Emfietzoglou, and R. Garcia-Molina, Nucl. Instrum. Methods Phys. Res., Sect. B 268, 1763 (2010).

[37] B. Senger and R. V. Rechenmann, Nucl. Instrum. Methods Phys. Res., Sect. B 2, 204 (1984).

[38] G. H. Olivera, P. D. Fainstein, and R. D. Rivarola, Phys. Med. Biol. 41, 1633 (1996).

[39] R. H. Ritchie and A. Howie, Philos. Mag. 36, 463 (1977).

[40] A. Salin, J. Phys. B 2, 631 (1969).

[41] F. A. Cucinotta, J. W. Wilson, R. Katz, W. Atwell, G. D. Badhwart, and M. R. Shave, Adv. Space Res. 18, 183 (1996).

[42] Z. Tan, Y. Xia, M. Zhao, X. Liu, F. Li, B. Huang, and Y. Ji, Nucl. Instrum. Methods Phys. Res., Sect. B 222, 27 (2004).

[43] L. C. Emerson, M. W. Williams, I. Tang, R. N. Hamm, and E. T. Arakawa, Radiat. Res. 63, 235 (1975).

[44] Y. Iriki, Y. Kikuchi, M. Imai, and A. Itoh, Phys. Rev. A 84, 032704 (2011).

[45] F. Salvat, J. M. Fernández-Varea, and J. Sempau, PENELOPE-2011: A Code System for Monte Carlo Simulation of Electron and Photon Transport (OECD/ NEA, Barcelona, 2011). 